\title{
Study to Determine the Outcomes of Closed Tibial Shaft Fractures Treated with Reamed Inter Locking Nails
}

\author{
SYED USMAN SHAH ${ }^{1}$, MUHAMMAD TARIQ KHAN ${ }^{2}$, ABDUL RASHEED NAPAR ${ }^{3}$, NASEER ULLAH KHATTAK ${ }^{2}$, AMINA GUL SHEHZAR \\ $\mathrm{KHAN}^{2}$, SAMI ULLAH ${ }^{2}$ \\ ${ }^{1}$ Assistant Professor Ayub Teaching Hospital, Abbottabad \\ ${ }^{2}$ TMO Orthopedics Ayub Teaching Hospital, Abbottabad \\ ${ }^{3}$ Assistant Professor Orthopedics Surgery, Khairpur Medical College, Khairpur Mir's Sindh \\ Corresponding author: Syed Usman Shah, Email: sust27@hotmail.com, Cell: +92 3132027777
}

\begin{abstract}
Aim: The aim of the study is to evaluate the results of treatment of a closed reamed interlocking in the treatment of closed fractures of the tibial shaft.

Study design: A Descriptive observational study

Venue and Duration: This study was conducted in the Orthopedic department of Ayub Teaching Hospital, Abbottabad for six months duration i.e from September 2020 to February 2021.

Patients and methods: The study included 43 patients over 19 years of age. The patients were taken to the emergency and Orthopedic department for surgery of Ayub Teaching Hospital, Abbottabad for six months duration i.e from September 2020 to February 2021. Written approvals were granted from all selectees. Detailed history was asked and each patient was assessed clinically and radiographically. The reamed intramedullary nailing performed according to the protocol. Patients were followed for 1 year and assessed for infection, union, knee and ankle range of motion, and implant problems. 3 patients were excluded from follow-up, and the study was held in 40 patients.

Results: All fractures were having complete union without the need for bone grafting. Simple fractures have union at mean of 14 weeks, comminuted and segmented fractures have union for longer than five weeks, and a mean duration of 18.5 weeks. Superficial infection at the screw site occurred in two patients, and the drainage was done, antibiotics were given and distal screw were removed, respectively. While all patients had a full range of knee motion, 37 patients had a full range of ankle movement. The other three patients had a 15-to-20-degree dorsiflexion loss at the ankle joint. No nail fracture, proximal screw end fracture in one patients and distal screw fracture in two patients were observed.

Conclusion: We came to the conclusion that closed intramedullary nailing in the case of a closed shaft fracture of the tibia is a safe and satisfactory technique with a high rate of union and a relatively low complication rate and early return to activity.

Keywords: Fracture, Internal, Fixation, Interlocking nail.
\end{abstract}

\section{INTRODUCTION}

The tibia is the most common long broken bone in the body due to its anatomical location and susceptibility to injury ${ }^{1-2}$. Treatment has always been a challenge for orthopedic surgeons due to the specific local anatomical feature, the high degree of fragmentation and the accompanying soft tissue trauma, which contributes to the high complication rate after open surgery ${ }^{3-4}$. The different treatment options include closed reduction and plaster of Paris, open reduction and internal fixation with plates and screws, external fixation, and intramedullary devices. POP cast has been associated with a high incidence of ankle stiffness. ${ }^{5}$ Plating are characterized by a high incidence of infection, non-union, and fixation failure ${ }^{6}$. External fixation has led to nail infection and sometimes osteomyelitis. A closed locking nail has been developed to reduce postoperative infections, promote early attachment and restore early activity ${ }^{7-8}$. The intramedullary nail is now widely used for the treatment of closed shaft fractures and is considered the preferred treatment for closed shaft fractures. The published series on the subject are extensive, with welldocumented high association rates associated with low complication rates. Malunion and malrotation have lower rates than another treatments $s^{9-10}$. These encouraging results have led to the use of widely reamed interlocking nails for the treatment of closed tibia fractures. Interlocking nails act as an inner splint that acts as a load-bearing tool and provides a bone graft at the fracture site. It provides power, not allowing the fracture to exceed a critical value that can disrupt the place of the fracture. Interlocking nails allow the adjacent joints to move, so rehabilitation is simultaneous with treatment and shear stresses are minimal.

\section{MATERIAL AND METHODS}

The study was conducted on 43 patients with closed fractures of the tibia who were admitted to the Orthopedic department of Ayub Teaching Hospital, Abbottabad for six months duration i.e from September 2020 to February 2021. All patients were assessed in the emergency and orthopedic department for risk of death according to the ATLS protocol. The fractures were splinted to lessen pain and prevent further soft tissue injury. Anterior-posterior and lateral X-rays, including the knee and ankle joints, were taken in all cases. The study included patients over 19 years of age with closed fractures of the tibia shaft, while patients with open and pathological fractures were excluded. Written approvals were granted from all selectees. In particular, a detailed history of the mechanism of the injury was requested, and a careful physical examination and appropriate tests were performed, including $\mathrm{HBsAg}$ and anti-HCV. The intramedullary nailing was performed as follows

Nailing Technique: All patients were operated on under GA on a regular operating table and $\mathrm{C}$-arm, with a pneumatic tourniquet on the mid of thigh and suspending their knees on the end of the table. A vertical midline incision was made from the lower pole of the patella to the tuberosity of the tibia. The patella tendon was retracted using a self-clamping retractor. Entry made $1.5 \mathrm{~cm}$ below the joint line with a curved awl in the center line. After entering the medullary cavity, a guide wire with an olive tip was inserted into the medullary cavity. A close reduction was made and the guidewire passed through the distal portion. When the guide passes through the distal part, the bone is palpated, which is confirmed under the $\mathrm{C}$ arm in both planes. Upon successful insertion of the guide, the tibia was reamed with an olive tipped wire using a flexible reamer. The guide with an olive tip was replaced with the help of a Teflon sleeve without losing the reduction and with a non-tipped guide wire. The length of the nail was assessed by subtracting the exposed length of the guide wire from the total length of the guide wire. The required nail is then inserted into the insertion assembly and then slides over the guide wire without hitting. The last part required light hammering. Once the nail had passed through the distal portion, the Guide wire was removed. All the nuts on the template were tightened securely. Limb traction was reduced and the foot was pushed back to push the distal proximally to compress the fracture site. The distal locking screws were then inserted using the free hand technique and the proximal screw was installed. The wound was closed without drainage. The knee is 
brought to its full range of motion. The patient was given intravenous antibiotics and painkillers. On the first postoperative day, a range of physical exercises was started, and the patient was mobilized using weighted crutches. The patients were discharged in the third postoperative period and 2 weeks later were reviewed. Later, patients were invited for another appointment at 1-month intervals until complete recovery. At each follow-up visit, patients were assessed for infection, union, range of motion of the knee and ankle, deformity, and any implant-related problems, and the results were documented on a previously prepared Proforma. 3 patients were excluded from follow-up, and the study was held in 40 patients

\section{RESULTS}

Thirty-four $(85.0 \%)$ of the 40 patients with an average age of 34 $(19-60)$ are men, and $6(15.0 \%)$ are women. The right side was affected in $29(72.5 \%)$ patients and the left side was affected in 11 $(27.5 \%)$ patients. Most of the fractures were segmental or sufficiently fragmented.

Table-I shows the demographic features of the patients

\begin{tabular}{|l|l|l|}
\hline Males & $34(85 \%)$ & 0.005 P-value \\
\hline Females & $6(15 \%)$ & 0.003 \\
\hline Laterality of the Fracture & \\
\hline Right & $29(72.5 \%)$ & 0.07 \\
\hline Left & $11(27.5 \%)$ & 0.04 \\
\hline
\end{tabular}

Fractures were classified according to the Winquist and Hansen classification and are shown in Table-II

\begin{tabular}{|l|l|l|}
\hline \multirow{2}{*}{ Winquist Type } & Number of & \multirow{2}{*}{ Percentage } \\
\cline { 2 - 3 } & Patients & \\
\hline I & 5 & $12.50 \%$ \\
\hline II & 9 & $22.5 \%$ \\
\hline III & 26 & $65 \%$ \\
\hline
\end{tabular}

Twenty fractures resulted from road accidents, 8 from significant falls, and 2 from sports injuries. The mechanism of the injury is shown in Table-III. 33 fractures were isolated, and 7 fractures were associated with an ipsilateral or contralateral femoral fracture, which was treated with a statically locked nail.

Table-III shows the various causes of fractures

\begin{tabular}{|l|l|l|}
\hline \multicolumn{2}{|l|}{ Causes of fractures } & P-value \\
\hline RTA & $27(67.5 \%)$ & 0.05 \\
\hline Falls & $9(22.5 \%)$ & 0.04 \\
\hline Sports Injuries & $4(10 \%)$ & 0.02 \\
\hline Isolated Fractures & $33(82.5 \%)$ & 0.06 \\
\hline $\begin{array}{l}\text { Ipsilateral or } \\
\text { contralateral } \\
\text { associated fractures }\end{array}$ & $7(17.5 \%)$ & 0.03 \\
\hline
\end{tabular}

All fractures were treated within the first week after injury and allowed for early mobilization. Patients were followed for 1 year and assessed for union (clinical and radiological), range of motion of the knee and ankle joint, infection, defective return, and implant problem. Fusion was achieved in all patients without the need for bone grafting.

$32(80 \%)$ fractures have union at 16-20 weeks (median 17 weeks) and 8 (20\%) occurred at median 13.5 weeks. Simple fractures have union at mean of 14 weeks, comminuted and segmented fractures have union for longer than five weeks, and a mean duration of 18.5 weeks. While $37(92.5 \%)$ fractures were locked statically, $3(7.5 \%)$ fractures were dynamically locked with proximal or distal screws. Between 6 and 9 weeks, 3 fractures were dynamized by removing the proximal or distal screw. Superficial infection at the screw site occurred in three patients, and the drainage was done, antibiotics were given and distal screw were removed, respectively. Six months later, two patients who developed an infection at the proximal or distal site of the screw was cultured with Staphylococcus aureus, and one patient 9 months later with Staphylococcus aureus in the distal part of the screw. Both patients were treated with drainage and antibiotics. All patients had a full range of motion in the knee joint (mean 140 degrees flexion). While the ankle joint showed that $37(92.5 \%)$ patients had full range of motion, $3(7.5 \%)$ patients had a 15-to-20degree dorsiflexion or plantar flexion reduction due to the primary foot injury. While none of the patients had a nail fracture, proximal screw end fracture occurred in 1 patient $(2.5 \%)$ and distal fracture occurred in 2 patients (5\%). Nothing has been done and the fractures have come together without incident.

\section{DISCUSSION}

Although a closed shaft fracture of the tibia can be treated nonsurgically, there are many indications for surgery. The preferred method of surgical treatment is controversial, but a chiseled, blocked intramedullary nail for closed shaft fracture of the tibia is a popular technique due to good results reported with relatively low complication rates in clinical surgical series ${ }^{11-12} \mathrm{~A}$ review of the literature on the subject shows that many authors use rates of union and infection as outcome criteria and ignore other important factors such as the range of motion of the upper and lower joints, problem with implants, and any deformities. We achieved $100 \%$ fusion without having to perform any other procedures such as bone grafting. In our study, the mean time to heal in patients with commuted or segmental fractures of the tibia was 18 weeks; This has been described by Court-Brown et al., Larsen et al. Comparable to the work done by. Lin et al. The infection rate in our series was low and delayed. Two patients developed an infection at the site of tuberosity secondary to the injury and was superficial. $\mathrm{He}$ was drained and treated with intravenous antibiotics. In the ninth month of the one patient who developed an infection in the distal screw area was removed, scraped and treated with antibiotics. In both cases, the cause is Staphylococcus aureus. Neither patient influences the final result. While all patients achieved full range of motion, 3 patients $(7.5 \%)$ had a reduced range of motion in the ankle by 15 to 20 degrees following the primary foot injury. Bonnevialle et al. He also found a normal range of motion in the knee and ankle joints ${ }^{15-16}$. The good range of motion in our series was due to the availability of a well-equipped physiotherapy unit. The main advantage of nesting seems to be the greater mobility it allows. The patient does not need a plaster cast, which facilitates joint movements and mobilization of the patient. The increased mobility provided by indoor diving facilitates an early return to all activities ${ }^{17}$. Because we carefully positioned the proximal and distal screws, no rotational deformation was observed in any of our patients ${ }^{18}$. Correct positioning of the proximal and distal screws almost completely eliminates shortening, rotating and angular deformations. Undoubtedly, the main problem with closed intramedullary nailing is undoubtedly the pain in the front of the knee, especially in our society where patients kneel to pray 5 times a day ${ }^{19}$. This problem can be prevented if trauma to the patellar tendon during impaction is minimized. Correct, stress-free skin closure also prevents the possibility of hypertrophic scarring and thus reduces pain at the front of the knee ${ }^{20}$. In our study, $55 \%$ patients had pain in the anterior part of the knee, Bonnevialle et al. A Vaisto et al study; $67 \%$ patients had knee pain, respectively ${ }^{21-22}$. We observed screw fracture in three patients. The proximal screws were broken in 2 patients and the distal screws in 1 patient. No specific action was taken and the failure did not jeopardize the end result. Other authors report an increased incidence of damage to locking screws associated with a non-grooved nail.

\section{CONCLUSION}

We came to the conclusion that closed intramedullary nailing for closed shaft fracture of the tibia is a safe and satisfactory technique with relatively low complication rates and high healing with early recovery. 


\section{REFERENCES}

1. Hendrickx LA, Virgin J, van den Bekerom MP, Doornberg JN, Kerkhoffs GM, Jaarsma RL. Complications and subsequent surgery after intra-medullary nailing for tibial shaft fractures: Review of 8110 patients. Injury. 2020 Jul 1;51(7):1647-54.

2. Bhan K, Tyagi A, Kainth T, Gupta A, Umar M. Reamed exchange nailing in nonunion of tibial shaft fractures: a review of the current evidence. Cureus. 2020 Jul;12(7).

3. Karmakar NC, Rahman MM. "Study of Management of Closed Tibial Shaft Fractures with Interlocking Nail under C-arm Guidance" Our experience in 250 Bedded General Hospital. The Journal of Bangladesh Orthopaedic Society (JBOS).:75

4. Whiting PS, Galat DD, Zirkle LG, Shaw MK, Galat JD. Risk factors for infection after intramedullary nailing of open Tibial shaft fractures in low-and middle-income countries. Journal of orthopaedic trauma. 2019 Jun 1;33(6):e234-9.

5. k Singh A, Sait S, Khan Y, Al-Obaidi B, Bhattacharya R. Suprapatellar nailing for isolated closed tibial shaft fractures: Medium term functional outcomes from an Academic Level 1 Trauma centre. Injury. 2020 Jul 1;51(7):1642-6

6. Basaran $T$, Calbiyik M, BASARAN PÖ, Hassa E, Ipek D. Blade expandable intramedullary nails for fixation of tibial shaft fractures. Acta Orthopædica Belgica. 2019 Dec 1;85:472-6.

7. Hsu KL, Kuan FC, Chang WL, Liu YF, Hong CK, Yeh ML, Su WR. Interlocking nailing of femoral shaft fractures with an extremely narrow medullary canal is associated with iatrogenic fractures. Injury. 2019 Dec 1;50(12):2306-11.

8. AKHTAR M, KHAN AF, GILLANI SF. Unreamed Tibial Nailing in Open Tibial Shaft Fractures. Age.;49(42.5):57-.

9. Khaleque A, Aslam MM, Islam MN. Evaluation of the Outcome of Tibial Shaft Fracture by Close Reduction and Internal Fixation with Interlocking Intramedullary Nail.

10. Alemdaroğlu KB, İltar S, Ozturk A, Gültaç E, Yücens M, Aydoğan NH. The role of biplanar distal locking in intramedullary nailing of tibia shaft fractures. Archives of Bone and Joint Surgery. 2019 Jan;7(1):33.

11. Patel J, Patel V, Oakley J, Sabharish CS, Reddy D, Jain A, Mani C, Desai SM. Study of interlocking nail in shaft tibia fracture International Journal of Orthopaedics. 2019;5(2):354-8.
12. Odatuwa-Omagbemi DO. Open intramedullary nailing using 'Greens Instrumentation'in the treatment of femoral and tibial shaft fractures: a 5 years review of cases in a private health facility in the Niger Delta region of Nigeria. Journal of Medicine in the Tropics. 2020 Jul $1 ; 22(2): 115$.

13. Hasan $\mathrm{MH}$, Hossain MA, Haque ME, Ahsanuzaman M, Parveen $\mathrm{E}$ Azad AK. Evaluation of Results of Closed Reamed Nailing for Diaphyseal Fracture of the Tibia-Experience of 35 cases. TAJ: Journal of Teachers Association. 2019 Aug 20;32(1):9-16.

14. Kostic I, Mitkovic M, Mitkovic M. The diaphyseal aseptic tibial nonunions after failed previous treatment options managed with the reamed intramedullary locking nail. Journal of clinical orthopaedics and trauma. 2019 Jan 1:10(1):182-90.

15. Namsha BR, Meghnathi UH, Hasan AN. Result of closed interlocking intramedullary nail in tibial shaft fracture. Knee.;22(3):13-04.

16. Khichy $H$, Garg A, Singh D, Mehta S. Outcome of antibiotic impregnated intramedullary nails in compound tibial fractures. International Journal of Orthopaedics. 2019;5(4):184-7.

17. Gathen M, Kabir K, Burger C. Segmental Tibia Shaft Fractures-IM Nailing. InFracture Reduction and Fixation Techniques 2020 (pp. 341-350). Springer, Cham.

18. Jain RK, Khare R, Jain N, Deshpande M. Study of management of distal $1 / 3$ rd tibial shaft fracture by intramedullary nailing.

19. Babu PA, Sudhakar K, Neal S, Rao DV, Kumar CS, Rao AS Prospective Study on Unreamed Interlocking Nail for Open Tibial Fractures.

20. Laigle $\mathrm{M}$, Rony $\mathrm{L}$, Pinet $\mathrm{R}$, Lancigu $\mathrm{R}$, Steiger $\mathrm{V}$, Hubert L. Intramedullary nailing for adult open tibial shaft fracture. An 85-case series. Orthopaedics \& Traumatology: Surgery \& Research. 2019 Sep 1;105(5):1021-4

21. Agarwala A, Kwan S, Matullo K. Esmarch technique for maintaining reduction during intramedullary nailing of tibial shaft fractures. Trauma case reports. 2019 Oct 1;23:100237.

22. Ullah R, Hussain A, Iqbal J, Imran A, Saddiq S, Ahmed N. Frequency of Union in Open Tibia Fractures treated with Unreamed Intramedullary Interlocking Nails. Journal of Pakistan Orthopaedic Association. 2020 Sep 10;32(02):87-91. 